\title{
Dscam-mediated Self- versus Non-Self-Recognition by Individual Neurons
}

\author{
G. Neves AND A. Chess \\ Center for Human Genetic Research, Department of Medicine, Massachusetts General Hospital and Harvard \\ Medical School, and Whitehead Institute for Biomedical Research, Cambridge, Massachusetts 02142
}

Clearly, gene regulation (the turning on and off of the multitudinous genes in the genome) allows the specification of different parts of the nervous system as it allows specification of all parts of the developing animal. Even within a group of neurons, distinguishing similar neurons also can be accomplished by the specific turning on and off of various genes via differential activities of various transcription factors. Moreover, differences in levels of expression of different genes and both regulated and stochastic aspects of posttranscriptional and posttranslational events are all important.

Alternative splicing represents another important mechanism that can render distinct cell populations different from one another. The mechanisms regulating the generation of alternative mRNA transcripts are known to have tissue specificity, and elucidation of this type of regulation is an important area of exploration. The generally accepted concept is that tissue-specific accessory splicing factors bind to cis-acting elements in the primary transcript and thus regulate the relative levels of different possible alternative splicing choices.

The alternative splicing of the Down syndrome cell adhesion molecule (Dscam) gene in Drosophila represents a particularly striking example of extensive alternative splicing with 38,016 possible splice forms (Schmucker et al. 2000): exon 4 has 12 possible forms; exon 6 has 48 possible forms; and exon 9 has 33 possible forms. Each of these three alternative exons specifies a portion of an immunoglobulin domain in the extracellular region of the protein. The variable exons are quite divergent (as low as $22 \%$ identity at the amino acid level) so that the encoded immunoglobulin domains are predicted to have very different three-dimensional structures. The mammalian Dscam has been shown to have homophilic interactions in vitro (Agarwala et al. 2000); this had earlier led to the idea that the different Dscam isoforms will differ in their interactions (Brummendorf and Lemmon 2001). In fact, recent elegant biochemical experiments of Zipursky and colleagues (Wojtowicz et al. 2004) have suggested that each one of the 19,008 different extracellular domains of Dscam may be able to recognize each other specifically. This represents a specificity of interaction reminiscent of the exquisite specificity of the antibody-antigen interaction with the added interesting twist that the recognition molecule recognizes itself. Prior to our work, while the exact specificity of individual Dscam isoforms had not been studied, prevailing hypotheses suggested that the extraordinary diversity would be used to distinguish different types of neurons from one another. Having each distinct neuronal cell type express a restricted portion of the repertoire would contribute to the different characteristic properties of the different cell types. This type of model emanated from the prevailing thinking in the field regarding the specification of cellular properties through the transcriptional regulation of the genome. As detailed below, our experiments lead us to an unanticipated and striking finding that Dscam alternative splicing allows a stochastic aspect to the generation of diversity of neurons. We find that while different populations of neurons have few limitations on choice and are therefore probably not distinguished by Dscam, at the level of individual neurons things become more interesting: Each individual neuron appears to be different from its neighboring cells by virtue of expression of very different Dscam isoforms. This allows each neuron to know when a portion of its membrane is contacting itself as opposed to contacting an adjacent neuron (that could be identical except for the Dscam difference). This striking self- versus non-self- awareness, at the single-cell level, allows the possibility that two adjacent cells, with theoretically identical levels of transcription of every gene in the genome, are distinct from one another.

\section{EXPERIMENTS ESTABLISHING THE DISPOSITION OF Dscam SPLICE FORMS}

When we began our studies, the existence of 38,016 possible alternative splice forms of Dscam posed the question of whether Dscam expression can provide a molecular signature of a distinct subpopulation of cells. The inordinately large number of possible alternative splice forms made it technically difficult to use standard methods of analyzing expression patterns such as in situ hybridization, immunohistochemistry, and transgenic animals. Therefore, to circumvent these technical obstacles, we developed an approach combining a customized oligonucleotide microarray with a sensitive reverse transcriptase polymerase chain reaction (RT-PCR) assay that allowed us to examine not only distinct populations of cells, but also single cells. The ability to examine single cells turned out to be critical to discovering the way in which Dscam diversity is used in neuronal specification. 
The experiments we carried out on individual cells have been published elsewhere (Neves et al. 2004) and will be summarized here. We designed a microarray containing probes for all 93 alternative exons in the three clusters that encode the variable extracellular immunoglobulin domains (exons 4, 6, and 9). Using the microarray, we first analyzed whole flies at various developmental time points. These initial experiments showed that splicing of Dscam is developmentally regulated, particularly with respect to exon 9 . For example, 5 of the 33 exon 9 isoforms are expressed at significantly higher levels in embryos than at the larval stages. Our results also confirm previous observations that the relative representation of exon 4.2 increases from embryos to adults (Celloto and Graveley 2001).

We then turned to the question of how the Dscam diversity is used, asking whether subsets of Dscam isoforms define different cell types. This was an intriguing possibility regarding the regulation of alternative splicing of the Dscam gene: that particular cell populations would each express a specific small subset of all the available isoforms. We analyzed a variety of cell types to search for significant differences in use of the Dscam repertoire by a number of different types of neuronal and nonneuronal cells. For example, we used the GAL4-UAS system (Brand and Perrimon 1993) to label specific populations of cells in the developing eye, and showed that both R3/R4 and R7 populations of photoreceptors express a large number of different isoforms at all three exon clusters. Each type of photoreceptor still expressed a repertoire of Dscam forms that was calculated to have a complexity well over 10,000 different forms (out of $\sim 19,000$ possible combinations for the extracellular diverse exons 4,6 , and 9). These results ruled out the model in which a specific class of photoreceptor that projects to a particular brain region expresses one or a small number of distinct Dscam isoforms. The observed broad spectra notwithstanding, clustering analysis indicates that the profiles of Dscam isoforms expressed by the two populations are significantly different from each other and from the entire eye-antennal imaginal disk. Thus, each specific cell type expresses a broad yet distinctive spectrum of Dscam isoforms. The differences in repertoire usage in the distinct cell populations may be functionally important, but more likely are not the primary reason for having such distinct repertoires. The likely reason for the enormous repertoire of Dscam emerged only from our single-cell analyses (see below).

The use of the microarray also allowed us to examine Dscam expression in S2 cell lines and subclones thereof. A very striking profile of Dscam isoforms is expressed in $\mathrm{S} 2$ cell lines. While the usage of different isoforms of exons 4 and 6 is roughly equal, for exon 9 apparently only 5 out of the 33 isoforms are used. Thus, there is a somewhat restricted repertoire of Dscam forms present within the population of S2 cells. Similar profiles were also observed with two other cell lines that have, like S2 cells, been suggested to share similar properties with hemocytes. Hemocytes isolated by fluorescence-activated cell sorter (FACS) from third-instar larvae that express green fluorescent protein (GFP) under the control of the promoter region of the hemocyte marker hemolectin (Goto et al. 2001) also show a similar profile. Also note that the same exon 9 isoforms are overrepresented in both whole embryos, larval eye-antennal disks, and larval hemocytes. Whether the use of these particular isoforms is functionally relevant, or whether they represent a "default" splicing mode that can be overwritten in more differentiated cells, remains an open question.

\section{SINGLE-CELL ANALYSES OF ALTERNATIVE SPLICING OF Dscam}

We developed and applied a sensitive and accurate RTPCR approach to determining the number of splice forms made by individual cells (Neves et al. 2004). The splitting of each single-cell RT reaction into multiple tubes allowed us to assess how robust the single-cell analysis was for each cell and to then employ statistical tests to arrive at firm conclusions regarding the disposition of the various splice forms in individual cells. Our first set of analyses included analyses of photoreceptor cells from the developing eye, and we also analyzed individual hemocytes. A number of important points emerged from our analyses. First, while a number of distinct Dscam transcripts were detected in each cell, there were not an extremely large number of transcripts detected. The range varied with the different cell types but allowed us to estimate that individual cells express in the range of 10 to 50 distinct mRNAs for Dscam at a given time. A second important point that emerged was the apparent randomness of the actual splice variants present in a given cell. These analyses indicate that a given cell's Dscam mRNA profile arises from a series of stochastic alternative splicing events for each Dscam transcript. The probability of selecting each individual alternative exon will be a function of the splicing factors expressed by each cell type. The choice of a small set of Dscam transcripts ( $\sim 50)$ from among many thousands of possible choices ensures that each individual neuron will be different from its neighbors, in terms of the Dscam repertoire that it expresses at its surface.

\section{SELF- VERSUS NON-SELF-DISCRIMINATION AT THE LEVEL OF INDIVIDUAL CELLS}

Our results suggest that each transcribed mRNA molecule will contain a novel combination of alternatively spliced exons, chosen from the broad spectrum of splice variants expressed by a given cell type. Since each cell expresses far fewer transcripts $(\sim 50)$ than the number of possible splice variants ( 19,000 extracellular forms), this results in every cell containing a Dscam repertoire that is different from those of its neighbors. The generation of a unique profile of cell surface protein isoforms in each cell through alternative splicing is an intriguing potential mechanism of specifying cell identity in the nervous system. It is conceivable that each cell could know itself as distinct from any other contacted cell solely by "reading" the contacted cell's pattern of Dscam isoforms in the context of its own Dscam repertoire. It is also possible that diverse cell compartments are generated using localized 
protein synthesis and mRNA localization mechanisms.

This concept is a type of self- versus non-self-distinction that has not been previously appreciated in the nervous system. Of course, in the immune system there are the well known examples where the expression of distinct $\mathrm{T}$ cell receptors by individual $\mathrm{T}$ cells allows each $\mathrm{T}$ cell a unique identity. Similarly, immunoglobulins on the surface of B cells afford unique identities to these cells. The unique identities of $\mathrm{T}$ and $\mathrm{B}$ cells are critical for the ability of these immune system cells to communicate with each other and coordinated the immune response. The concept of self versus non-self in the case of the immune system is referring to the complex ability of an "educated" $\mathrm{T}$ cell to distinguish cells from its own body as different from transplanted or infected cells. The unique identity given to each $\mathrm{T}$ cell by its T-cell receptor is central to the proper functioning of the immune system. It is interesting to note that Dscam alternative splicing provides an even greater difference between otherwise similar cells because, after cell division, while two daughter T cells will now share the same T-cell receptor, two daughter Dscam-expressing cells will over time elaborate distinct repertoires of Dscam alternative splice forms.

An interesting emerging model for how self- versus non-self-distinction is used by neurons invokes a dual function for Dscam. In this model-which is supported by studies of mushroom body neurons we published as part of a collaborative effort with Zipursky and colleagues that included genetic data (Zhan et al. 2004), as well as genetic studies of T. Lee and colleagues (Wang et al. 2004) - the first function is a homophilic adhesivity based on the overall structure common to all Dscam isoforms. In other words, irrespective of the exact isoform, any two molecules of Dscam whose extracellular domains encounter each other (in the correct orientation relative to each other) will have an adhesive interaction. This function is similar to the function ascribed to the mammalian Dscams (Barlow et al. 2002). The key to the model is the second function of Dscam. The second function is an ability to transduce a signal that leads to repulsion if two identical Dscam isoforms interact. It is possible that the triggering of the repulsive interaction could be accomplished by the strength of the adhesion or by some other signal transduction that can occur only when two identical Dscam molecules interact. This model is in certain ways reminiscent of antigen recognition by $\mathrm{T}$ cells especially during thymic education; the use of the strongest of interactions to mediate a negative signal has been observed in T-cell receptor interactions with peptide antigens presented in the context of the major histocompatibility complex (MHC).

Let us now consider how this model would play out when two portions of membrane touch one another. In the normal situation, two portions of membrane from two distinct axons would contact one another; Dscam molecules on each membrane would sense the presence of Dscam on the other membrane; and, then, through a combination of simple adhesion and perhaps a positive signal activating other adhesivity molecules, the Dscam interaction would promote fasciculation of the two axons.
Importantly, the sensing of the Dscam in this model would include a determination, as it were, of the fact that the two Dscam molecules are different from one another. In the unlikely event that the exact matching Dscam were to be present on the two interacting axons, then a negative signal would be generated, thus promoting repulsion.

Now consider the expectation under various abnormal settings. In the absence of Dscam the expectation is relatively straightforward: Without the Dscam interaction, the portions of membrane will not have the Dscam-mediated adhesive interaction (direct or indirect) and thus the axons will not fasciculate. In another scenario wherein the axons encountering each other have the same Dscam isoform, the second function of Dscam that can mediate a repulsive interaction wins the day and the axons will not fasciculate. To date, the available genetic studies (Wang et al. 2004; Zhan et al. 2004) are consistent with this model. The most striking data indicate that a Dscam null mutation can be rescued by a single form in a single-cell clone, but not in a larger clone. A single-cell clone expressing a single form of Dscam will be different from any cell it encounters and will therefore still be able to determine that it is different from its neighboring cells.

\section{IS SELF- VERSUS NON-SELF-DISCRIMINATION A MORE GENERAL PRINCIPLE?}

Extensive alternative splicing of Dscam orthologs is conserved in disparate insect species, and, while Dscam does not have extensive alternative splicing in mammals (Barlow et al. 2002), other cell surface protein families involved in neuronal development such as neurexins (Tabuchi and Sudhof 2002) and protocadherins (Wu et al. 2001) have numerous differentially spliced forms. The neurexins in mammals, for example, have well over 1000 possible forms and play roles in cell adhesion and synapse formation. Neurexins in flies and worms are much simpler and have little or no alternative splicing (Tabuchi and Sudhof 2002). The protocadherins also have an interesting complexity in mammals that is not found in fly homologs. Each protocadherin gene has a single constant region with a multitude of variable regions upstream of it. It appears that differential promoter usage leads to the observed expression of distinct combinations of isoforms in individual neurons. Whether the promoters are regulated like classical promoters, or through some alternative, stochastic mechanism remains to be determined. If stochastic regulation leads to the observed expression patterns, one would expect to observe the independent regulation of the two chromosomes leading to instances of monoallelic expression and instances of biallelic expression. Single-cell RT-PCR and microarray analyses of alternatively spliced isoforms could allow determination of the distribution of alternative isoforms for these other genes and their possible roles in specifying neuronal identity.

Each of these gene families that undergo extensive alternative splicing may be accomplishing two distinct functions. A "baseline" function would be whatever the gene is doing in the species in which it is not alternatively 
spliced. This baseline function would presumably need to be maintained even in the context of a second function of generating diversity at the cell surface of each individual neuron. This second function, a requirement for the generation of diversity, has been accomplished by increasing the splicing-mediated diversity of different genes in different types of organisms.

Any individual gene has to be looked at as one of many genes that influence any given behavior of a type of cell. The properties of neurons are especially complex. The significance of studying the alternative splicing of Dscam emanates from the extraordinary diversity of splice forms that are possible. Also adding to the significance is the pattern of usage of different splice forms by different cells that is beginning to emerge from our studies. Taken together with the fact that other genes such as neurexins may play similar roles in mammals, these studies will be an important component of an increasing understanding of the generation of an enormous amount of diversity in neuronal type in complex nervous systems. Upon the emergence of a more complete picture of the patterns of different splice forms displayed by different cells, we will be in a position to begin to examine the ways in which the enormous diversity is interplaying with other gene products in mediating specific properties of classes of neurons and individual neurons.

\section{ACKNOWLEDGMENTS}

We thank colleagues in the Chess laboratory for stimulating discussions. We are thankful for support of this work from the NIH (NINDS) to A.C.

\section{REFERENCES}

Agarwala K.L., Nakamura S., Tsutsumi Y., and Yamakawa K. 2000. Down syndrome cell adhesion molecule Dscam mediates homophilic intercellular adhesion. Brain Res. Mol. Brain
Res. 79: 118 .

Barlow G.M., Micales B., Chen X.N., Lyons G.E., and Korenberg J.R. 2002. Mammalian Dscams: Roles in the development of the spinal cord, cortex, and cerebellum? Biochem. Biophys. Res. Commun. 293: 881 .

Brand A.H. and Perrimon N. 1993. Targeted gene expression as a means of altering cell fates and generating dominant phenotypes. Development 118: 401.

Brummendorf T. and Lemmon V. 2001. Immunoglobulin superfamily receptors: cis-interactions, intracellular adapters and alternative splicing regulate adhesion. Curr. Opin. Cell Biol. 13: 611.

Celotto A.M. and Graveley B.R. 2001. Alternative splicing of the Drosophila Dscam pre-mRNA is both temporally and spatially regulated. Genetics 159: 599.

Goto A., Kumagai T., Kumagai C., Hirose J., Narita H., Mori H., Kadowaki T., Beck K., and Kitagawa Y.A. 2001. Drosophila haemocyte-specific protein, hemolectin, similar to human von Willebrand factor. Biochem. J. 359: 99.

Neves G., Zucker J., Daly M., and Chess A. 2004. Stochastic yet biased expression of multiple Dscam splice variants by individual cells. Nat. Genet. 36: 240.

Schmucker D., Clemens J.C., Shu H., Worby C.A., Xiao J., Muda M., Dixon J.E., and Zipursky S.L. 2000. Drosophila Dscam is an axon guidance receptor exhibiting extraordinary molecular diversity. Cell 101: 671.

Tabuchi K. and Sudhof T.C. 2002. Structure and evolution of neurexin genes: Insight into the mechanism of alternative splicing. Genomics 79: 849 .

Wang J., Ma X., Yang J.S., Zheng X., Zugates C.T., Lee C.-H.J., and Lee T. 2004. Transmembrane/juxtamembrane domain-dependent Dscam distribution and function during mushroom body neuronal morphogenesis. Neuron 43: 663 .

Wojtowicz W.M., Flanagan J.J., Millard S.S., Zipursky S.L., and Clemens J.C. 2004. Alternative splicing of Drosophila Dscam generates axon guidance receptors that exhibit isoform-specific homophilic binding. Cell 118: 619.

Wu Q., Zhang T., Cheng J.F., Kim Y., Grimwood J., Schmutz J., Dickson M., Noonan J.P., Zhang M.Q., Myers R.M., and Maniatis T. 2001. Comparative DNA sequence analysis of mouse and human protocadherin gene clusters. Genome Res. 11: 389.

Zhan X.L., Clemens J.C., Neves G., Hattori D., Flanagan J.J., Hummel T., Vasconcelos M.L., Chess A., and Zipursky S.L. 2004. Analysis of Dscam diversity in regulating axon guidance in Drosophila mushroom bodies. Neuron 43: 673. 


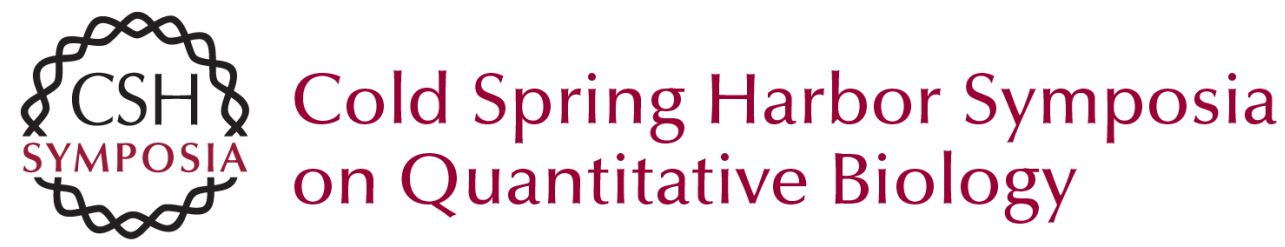

\section{Dscam-mediated Self- versus Non-Self-Recognition by Individual Neurons}

G. NEVES and A. CHESS

Cold Spring Harb Symp Quant Biol 2004 69: 485-488

Access the most recent version at doi:10.1101/sqb.2004.69.485

References This article cites 13 articles, 3 of which can be accessed free at: http://symposium.cshlp.org/content/69/485.full.html\#ref-list-1

License

Email Alerting Receive free email alerts when new articles cite this article - sign up in Service the box at the top right corner of the article or click here. 